\title{
UTILISATION DE LA MARGINE EN TANT QU'ADJUVANT DANS LE BÉTON
}

\author{
Faouzia Charfi Fourati*, Jamel Rouis** et Boubaker Elleuch ${ }^{* * *}$ \\ Enis (Tunisie)
}

\begin{abstract}
Dans ce travail, nous avons étudié d'une part l'influence du pourcentage de margine sur la fluidité du béton frais. Il s'est avéré que la mesure de l'affaissement du béton au cône d'Abrams augmente au fur et à mesure que le pourcentage d'ajout de margine augmente. D'autre part, nous avons étudié l'influence du pourcentage de margine sur l'évolution de la résistance à la compression et de la densité du béton. Nous avons remarqué que pour un pourcentage d'ajout n'excédant pas $0,2 \%$, la margine augmente la résistance à la compression du béton. Au delà de cette valeur, la résistance diminue. Concernant la densité du béton, celle-ci diminue proportionnellement avec le pourcentage d'ajout. L'ajout de margine au béton assure la neutralisation des charges électriques se trouvant à la surface des grains de ciment en s'adsorbant sur ces derniers. Ce phénomène d'enrobage est facilité par la forme linéaire des molécules de margine. Les grains de ciment enrobés ont tendance à s'éloigner les uns des autres étant donné qu'ils ont tous la même charge. Ainsi on obtient un gain de fluidité puisque les grains sont mieux dispersés et ne retiennent plus d'eau. Cette eau devenue libre sert alors à fluidifier le béton.
\end{abstract}

In this work, we studied the incorporated proportion of Olive Mill Waste Water on the measure of Slump test. That showed that subsidence of fresh concrete increases as the amount of Olive Mill Waste Water increased. The influence of the amount of this additive on the compressive strength and specific weight of concrete was investigated. We noticed for a percentage of Olive Mill Waste Water below 0,2 \%, the additive increase the compressive strength of concrete; beyond this value, the compressive strength decreases. Regarding the concrete's specific weight, it decreases when the rate of Olive Mill Waste Water increases by a linear function.

\section{INTRODUCTION}

En plus de l'huile comme produit principal, l'industrie d'huile d'olive produit de grandes quantités de sous-produits (grignon et margine).
La margine représente $50 \%$ du poids des olives traitées. Les résidus solides varient de 3 à $10 \%$. Les solides insolubles varient de 0,04 à $0,5 \%$.

La production annuelle de margine partout dans le monde varie de 25 à 40 millions de mètres cube. Dans le bassin méditerranéen, la production de margine varie de 20 à 35 millions de mètres cube par an; ce qui correspond à $90 \%$ de la production mondiale. Étant donnée l'énorme quantité de margine produite durant l'année, il est nécessaire de valoriser ce sous-produit de l'olive.

La composition de la margine varie avec le type d'olive et la méthode d'extraction de l'huile. Les valeurs moyennes en matières organiques et minérales (M.O. et M.M.) sont présentées dans le tableau $\mathrm{I}$.

Notre étude se concentre sur les effets de la margine (pro-

\begin{tabular}{|c|c|c|}
\hline \multicolumn{3}{|c|}{$\begin{array}{l}\text { Tableau I: Composition de la margine Tunisienne }{ }^{[3,5} \\
\text { et Espagnole }\end{array}$} \\
\hline Nefzaoui ${ }^{[3]}$ & $\begin{array}{l}\text { Fiestas ros } \\
\text { de ursinos }{ }^{[5]}\end{array}$ & Charfi Fourati ${ }^{[6]}$ \\
\hline Eau 83 \% & Eau 83 à 92 \% & Eau $90 \%$ \\
\hline $\begin{array}{l}\text { Matière organique } \\
\qquad 15 \%\end{array}$ & $\begin{array}{l}\text { Matière organique } \\
7 \text { à } 15 \%\end{array}$ & $\begin{array}{l}\text { Matière organique } \\
\quad 8,5 \%\end{array}$ \\
\hline $\begin{array}{l}\text { Matière minérale } \\
\qquad 2 \%\end{array}$ & $\begin{array}{l}\text { Matière minérale } \\
\text { I à } 2 \%\end{array}$ & $\begin{array}{l}\text { Matière minérale } \\
\quad \text { I,5\% }\end{array}$ \\
\hline
\end{tabular}

duit tunisien) sur la rhéologie et sur les caractéristiques mécaniques du béton.

\section{MATÉRIAUX ET MÉTHODES}

Dans la première étape, l'impact de l'addition de la margine sur la résistance à la compression du béton à différents âges a été étudié pour des concentrations différentes d'ajout.

Dans une deuxième étape, nous avons examiné l'effet de la margine sur la masse volumique du béton, et par conséquent sa porosité. Pour ce faire, des mélanges contenant différents dosages de margine ont été testés. Dans ces mélanges, le rapport eau/ciment a été gardé constant $(0,53)$. 


\section{CARACTÉRISTIQUES DES MATÉRIAUX UTILISÉS}

\section{Ciment}

Le ciment utilisé est un ciment Portland du type (II-A-L $32,5)$, contenant $9 \%$ de calcaire environ.

En se référant à sa composition chimique, la composition minéralogique du ciment a été calculée en utilisant les formules de Bogue-Dal (ASTM CII4) ${ }^{[7]}$. Cette composition minéralogique est présentée dans le tableau 2. Le ciment utilisé dans cette étude présente une perte au feu de $5,93 \%$ (à $1000^{\circ} \mathrm{C}$ ), une surface spécifique Blaine de $2620 \mathrm{~cm}^{2} / g$ et une masse volumique absolue de $3,07 \mathrm{~g} / \mathrm{cm}^{3}$. L'analyse granulométrique a été réalisée avec la méthode laser. Les résultats obtenus sont montrés par la figure $\mathrm{I}$.

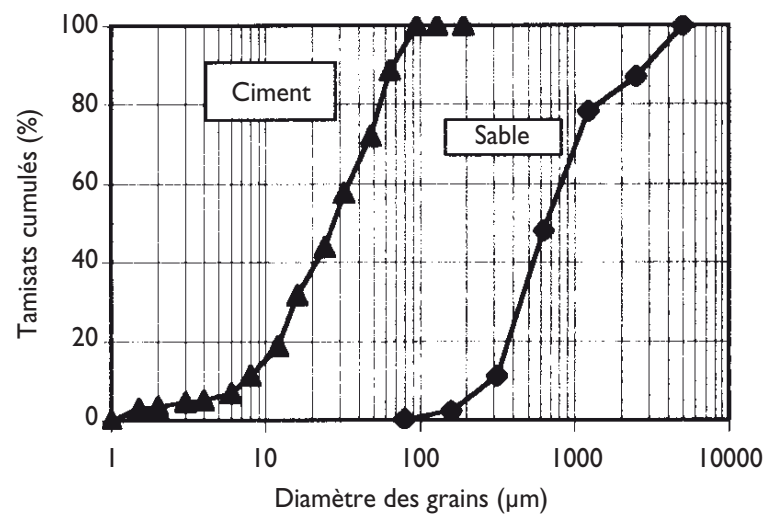

Figure I : Distribution granulométrique des particules de ciment et de sable

\section{Sable}

Le sable utilisé lors de cette étude est un sable d'oued de la région «Chaffar», située au sud de la ville de Sfax (Tunisie). La masse volumique absolue de ce sable est $2,62 \mathrm{~g} / \mathrm{cm}^{3}$. L'analyse granulométrique réalisée avec le tamiseur automatique a conduit aux résultats présentés par la figure I. Cette figure représente la variation des diamètres des grains de ciment et de sable en fonction des tamisats cumulés en $\%$. Le module de finesse du sable est égal à 2,7. L'équivalent de sable est égal à $52 \%$. Par conséquent, ce sable est considéré préférentiel.

\begin{tabular}{|c|c|c|c|c|}
\hline \multicolumn{2}{|c|}{$\begin{array}{l}\text { Tableau 2: } \\
\text { Composition } \\
\text { minéralogique } \\
\text { du ciment }\end{array}$} & \multicolumn{3}{|c|}{$\begin{array}{l}\text { Tableau 3: } \\
\text { Indice de fragmentation } \\
\text { dynamique du gravier }\end{array}$} \\
\hline $\begin{array}{l}\text { Élément } \\
\text { minéral (a) }\end{array}$ & Teneur & Échantillon & $\begin{array}{c}\text { Classe } \\
\mathbf{N}^{\circ}\end{array}$ & $\begin{array}{l}F_{D}{ }^{(b)} \\
\text { moyen }\end{array}$ \\
\hline «C $\mathrm{C}_{3} \mathrm{~S} »$ & 41,06 & Graviers 4-I5 & A & 30,73 \\
\hline " $\mathrm{C}_{2} \mathrm{~S}$ » & 21,19 & Graviers 4-15 & B & 28,68 \\
\hline " $C_{3} A$ ॥ & 9.19 & Graviers 4-I5 & C & 27,02 \\
\hline " $C_{4} A F$ » & 8,02 & Graviers $15-25$ & A & 30,26 \\
\hline $\mathrm{CaSO}_{4}$ & 3,01 & Graviers $15-25$ & B & 26,51 \\
\hline $\mathrm{CaCO}_{3}$ & $|7,6|$ & Graviers $15-25$ & C & 25,71 \\
\hline
\end{tabular}

\section{Gravier}

Deux classes de graviers ont été utilisées: $4-15$ et $15-25 \mathrm{~mm}$. Leurs indices de fragmentation dynamique sont présentés sur le tableau 3.

La masse volumique absolue du gravier mesurée avec le densimètre « Le Chatelier» est égale à $2,61 \mathrm{~g} / \mathrm{cm}^{3}$.

\section{Adjuvant}

L'adjuvant utilisé est la margine tunisienne ayant les caractéristiques suivantes:

- densité :I,07,

- $\mathrm{pH}: 5,3$,

- extrait sec ( déterminé d'après la norme NFP I8-380 ): $10 \%$.

La composition chimique de la margine est représentée sur les tableaux 4 et 5 .

\begin{tabular}{|c|c|c|c|}
\hline \multicolumn{2}{|c|}{$\begin{array}{c}\text { Tableau } 4 \text { : } \\
\text { Matière minérale }^{[9]}\end{array}$} & \multicolumn{2}{|c|}{$\begin{array}{c}\text { Tableau 5: } \\
\text { Matière organique }{ }^{[9]}\end{array}$} \\
\hline Élément & Teneur (ppm) & Élément & Teneur \\
\hline $\mathrm{P}_{2} \mathrm{O}_{5}$ & 310 & Sucres & $0,67 \%$ \\
\hline $\mathrm{K}^{+}$ & 9210 & Huiles résiduelles & $0,03-1 \%$ \\
\hline $\mathrm{Na}^{+}$ & 964 & Protéines & $0,05-0,75 \%$ \\
\hline $\mathrm{Ca}^{++}$ & 536 & Pectines & $0,6 \%$ \\
\hline $\mathrm{Mg}^{++}$ & 482 & Vitamines & 124 p.p.m. \\
\hline $\mathrm{Cl}^{-}$ & 4122 & Composés & \multirow{6}{*}{ I à $2,4 \%$} \\
\hline $\mathrm{Fe}^{3+}$ & 91 & Monoet & \\
\hline $\mathrm{SiO}_{2}$ & 5800 & Composés & \\
\hline $\mathrm{SO}_{4}^{-}$ & 1273 & Poly - aromatique & \\
\hline $\mathrm{Al}^{3+}$ & 22 & & \\
\hline $\mathrm{Zn}^{++}$ & 2 & & \\
\hline
\end{tabular}

Les analyses chimiques sont réalisées au Centre de biotechnologie de Sfax (CBS) et au Groupe chimique tunisien (GCT).

Les teneurs en phosphore, en chlorure et en sulfate sont déterminées par la méthode gravimétrique. Le potassium et le sodium sont déterminés par spectrophotométrie de flamme. La silice, le calcium, le magnésium, le fer, l'aluminium, et le zinc sont déterminés par spectrométrie d'absorption atomique. La quantité de sucres est déterminée par la méthode de Miller. Les composés mono-aromatiques sont déterminés par la méthode de Folin-Ciocalteau ${ }^{[8]}$. Les composés poly-aromatiques sont déterminés par la méthode HPLC.

\section{PROCÉDURES EXPÉRIMENTALES}

La résistance à la compression a été testée en utilisant une presse de compression de 2000 kN. Cela nous a permis d'obtenir une contrainte maximale de $89 \mathrm{MPa}$ mesurée sur éprouvettes cubiques ( $15 / 15$ centimètres).

La cure des éprouvettes a été réalisée à l'eau. Ces éprouvettes ont été retirées de l'eau deux heures avant l'essai de résistance à la compression. La masse volumique de l'échantillon de béton a été mesurée. Les dosages de margine utilisés étaient compris entre $0,1 \%$ et $2 \%$ et ils sont donnés 
en masse sèche.

La composition utilisée pour béton était la suivante:

$\mathrm{C}=378 \mathrm{~kg} / \mathrm{m}^{3}$

$\mathrm{S}=669 \mathrm{~kg} / \mathrm{m}^{3}$

$\mathrm{G}=1093 \mathrm{~kg} / \mathrm{m}^{3}$

$E=200 \mathrm{~kg} / \mathrm{m}^{3}$

Le gravier utilisé était pour moitié de la classe 4 - I5 et pour moitié de la classe 15-25.

L'eau de robinet utilisée contenait $100 \mathrm{mg} / \mathrm{l}$ de calcium et $55 \mathrm{mg} / \mathrm{l}$ de magnésium.

\section{RÉSULTATS}

\section{Effet de la margine sur la fluidité du béton frais}

La margine entraîne une augmentation de la fluidité du béton. En effet, en augmentant le pourcentage de margine tout en conservant un rapport eau/ciment constant, l'affaissement au cône d'Abrams augmente à un point tel que sa mesure devient impossible pour les pourcentages élevés (tableau 6).

Par ailleurs, un phénomène de ressuage est remarqué.

\section{Table 6: Essai d'affaissement}

\begin{tabular}{lcccccc} 
Pourcentage de margine & 0 & 0,1 & 0,2 & 0,3 & 1 & 2 \\
\hline $\begin{array}{l}\text { Mesure d'affaissement } \\
\text { au cône d'Abrams (cm) }\end{array}$ & 7,0 & 7,5 & 8,0 & 9,0 & 13,0 & -
\end{tabular}

Une pellicule fine d'eau, d'environ $2 \mathrm{~mm}$ d'épaisseur, apparaît à la surface des éprouvettes. Cette pellicule donne, après durcissement, une couche mince et fragile. Ce comportement est observé surtout avec des pourcentages élevés de margine (au delà de $0,3 \%$ ).

\section{Effet de la margine sur la résistance du béton}

Pour évaluer l'interaction de la margine avec le béton et comprendre son mécanisme d'action, la résistance à la compression de tous les matériaux préparés, a été déterminée à différents âges.

La figure 2 représente la variation de la résistance à la compression en fonction de l'âge pour différents pourcentages

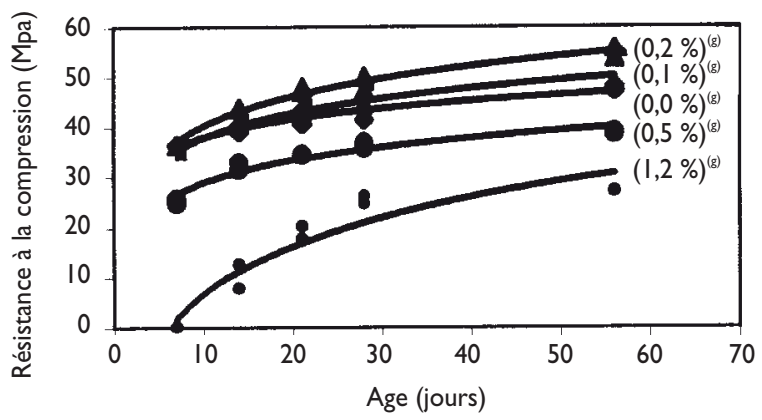

Figure 2: Variation de la résistance à la compression en fonction de l'âge pour différents pourcentages de margine de margine.

La figure 2 montre que:

- la valeur la plus grande de la résistance à la compression est enregistrée pour un dosage de margine de 0,2\%; et cela est vrai pour tous les âges ;

- quand le taux de margine dépasse $0,2 \%$, plus le pourcentage de margine est élevé plus la résistance à la compression est faible ;

- au-dessus de 0,2 \% de margine ajouté, le béton devient plus poreux parce que la masse volumique baisse (Figure 3); alors, la compacité et la résistance à la compression diminuent.

\section{Effet de la margine sur le poids spécifique du béton}

La figure 3 qui représente les variations de la masse volumique du béton en fonction du pourcentage de margine, montre que:

- la variation de la masse volumique est représentée par une fonction linéaire du pourcentage de margine;

- la masse volumique du béton diminue au fur et à mesure que le pourcentage de margine augmente. Cet effet a une grande importance dans le cas des ouvrages en béton armécar une diminution du poids de la pièce à confectionner implique un gain de ferraillage.

En plus, la résistance à la compression est contrôlée aussi par la variation de la masse volumique du béton. Les expériences réalisées ont montré une bonne corrélation entre ces deux paramètres. Cependant, une augmentation de la masse volumique est suivie systématiquement d'une augmentation de la résistance à la compression quel que soit l'âge du béton.

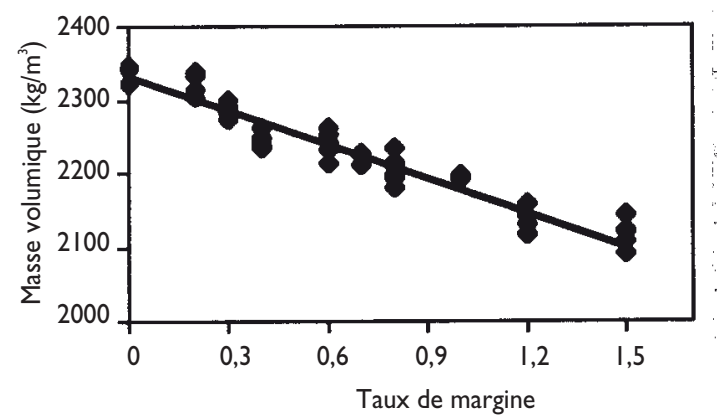

Figure 3 : Variation de la masse volumique du béton en fonction du pourcentage de margine ajouté

\section{DISCUSSION}

Une augmentation de la fluidité du béton a été enregistrée avec l'addition de margine. Pour maintenir une ouvrabilité constante du béton en présence de la margine, le rapport eau/ciment peut être diminué: la margine fonctionne alors comme réducteur d'eau. D'où l'augmentation de la résistance du béton; la margine agit par conséquent comme un agent fluidifiant.

Les fluidifiants sont généralement des tensioactifs anioniques, 
cationiques ou non-ioniques. Leur ajout au béton assure la neutralisation des charges électriques qui entourent les particules de ciment par adsorption sur ces derniers. Ce phénomène d'enrobage est facilité par la forme linéaire des molécules de fluidifiants. Les particules de ciment enrobés ont tendance à s'éloigner les unes des autres étant donné qu'elles ont toutes la même charge. L'eau libre qui n'est plus retenue par les particules de ciment participe également à l'augmentation de la fluidité du béton.

Les tensioactifs non-ioniques peuvent provoquer le même phénomène pour le béton et pour les coulis de ciment. Ils agissent comme agents fluidifiants et permettent de réduire la quantité d'eau nécessaire pour la fabrication du béton. Leur mode d'action est différent de celui des molécules tensioactives anioniques. Une description de ce mode d'action a été reportée par Aitcin*.

Le gain de fluidité obtenu avec la margine peut être expliqué par les phénomènes mentionnés ci-dessus et par la nature de quelques composants présents dans la margine. En effet, l'examen de la composition chimique de la margine permet de distinguer différentes molécules qui ont les propriétés des tensioactifs.

Dans la margine, les sels d'acides gras sont des tensioactifs anioniques. Les molécules polymérisées présentes avec des groupements glucosidiques donnent à l'ensemble de la molécule la structure de tensioactifs non-ioniques.

À titre d'exemple, on peut citer les molécules appartenant à la famille des glucosides phénoliques tels que l'anthocyane et les tanins (gallotannin).

\section{CONCLUSIONS}

Cette étude a montré les effets de la margine sur les caractéristiques de béton à l'état frais et durci. L'étude du comportement du béton adjuvanté à la margine a montré que: - Bien que le rapport eau/ciment soit gardé constant, une augmentation de la résistance à la compression pour un pourcentage de margine inférieur à $0,2 \%$ a été remarquée; - La masse volumique du béton diminue au fur et à mesure de l'augmentation de pourcentage de margine.

\footnotetext{
* Faouzia Charfi Fourati,

Docteur en Géologie-Laboratoire de chimie industrielle - ENIS - BP W 3038 Sfax (Tunisie)

\section{** Jamel Rouis,}

Professeur en Geologie-Laboratoire de géotechnique - ENIS - BP W 3038 Sfax (Tunisie)

\section{*** Boubaker Elleuch,}

Professeur en Chimie-Laboratoire eau-energie-environnement - ENIS BP W - 3038 Sfax (Tunisie)

* Sous cet acronyme se cache l'auteur de l'article intitulé « Les fluidisants : des réducteurs d'eau pas comme les autres» publié dans les Annales de I'ITBTP n 473 (mars-avril 1989, pp. I5I-155).
}

\section{Références}

I. G. Boari, A. Brunetti, R. Passino and 8. O. Folin, V. Ciocalteau, Journal of A. Rozzi, Agricultural Wastes, 1984, Biological Chemestry, 1927, p. 62710, p. I6I-175. 650.

$\begin{array}{ll}\text { 2. N. Gharsallah, Environmental Tech- } & \text { 9. F. Charfi Fourati, J. Rouis, B. Elleuch, } \\ \text { nology, 1993, I4, p. 39I-395. } & \text { Journal de la Société Chimique tuni- }\end{array}$ sienne, 200I, 4, $\mathrm{n}^{\circ} 9$.

3. A. Nefzaoui, Options Méditerranéennes, Série Séminaires, 1991, $n^{\circ} 16$, p. I0I - 108.

4. M. Karapinar and M.J.T. Worgan, J. Chem. Techn. Biotechnol., 1983, 33B, p. $185-188$.

5. F. Charfi Fourati, Utilisation de la margine en tant qu'adjuvant dans les coulis d'injection et dans les bétons, DEA de Géologie appliquée à l'environnement, Université de Tunis II, 1994.

6. J.A. Fiestas Ros de Ursinos, R. Navarro Gamero, R. Leon Gabello, A.J. Garcia Buendia and G.M. Mastrojuan Saez de Jauragui, Depuracion anaerobia del alpechin como fuente de energia, 1982.

7. Bogue, R.H., Industr. Eng. Chem. Anal., 1929, I, p. 192 - 197.
10. P.C. Aitcin, Annales de l'ITBTP, 1989, N 473, Mars-Avril, p. I5I-I55.

\section{Nomenclature}

(a): nomenclature chimique utilisée $\mathrm{C}=\mathrm{CaO}, \mathrm{S}=\mathrm{SiO}_{2}, \mathrm{~A}=\mathrm{Al}_{2} \mathrm{O}_{3}, \mathrm{~F}$ $=\mathrm{Fe}_{2} \mathrm{O}_{3}$

(b): $F_{D}$ : indice de fragmentation dynamique

(c): $C=$ ciment

(d) : $S=$ sable

(e): $G=$ gravier

(f): $E=$ eau

$(\mathrm{g}):(\mathrm{X} \%)=$ taux de margine

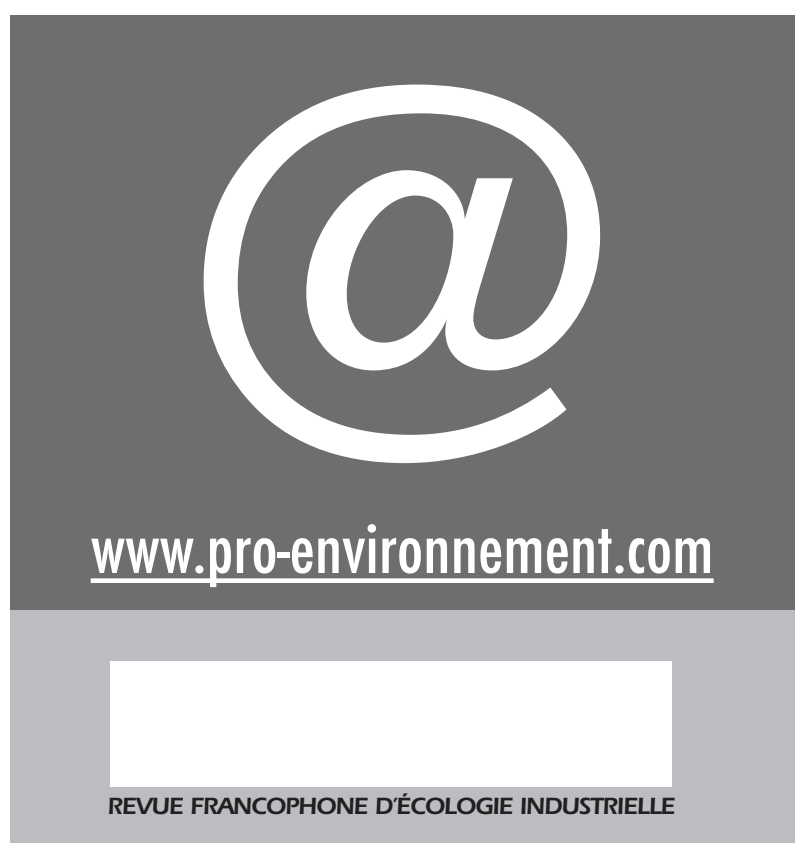

